\title{
Identification and antibiotic susceptibility of coagulase negative staphylococci isolated in corneal/external infections
}

\begin{abstract}
Antonio Pinna, Stefania Zanetti, Mario Sotgiu, Leonardo A Sechi, Giovanni Fadda, Francesco Carta
\end{abstract}

\begin{abstract}
Aims-To identify and determine antibiotic susceptibility of coagulase negative staphylococci (CoNS) isolated from patients with chronic blepharitis, purulent conjunctivitis, and suppurative keratitis.

Methods-A retrospective review of all culture positive cases of chronic blepharitis, purulent conjunctivitis, and suppurative keratitis between July 1995 and December 1996 was performed. Cases in which CoNS were the sole isolates were analysed. Species identification was performed by using a commercially available standardised biochemical test system. Antibiotic susceptibility to penicillin, gentamicin, tetracycline, erythromycin, ciprofloxacin, and teicoplanin was determined by agar disc diffusion (KirbyBauer method). Teicoplanin resistance was confirmed by agar dilution.
\end{abstract}

Results-42 Staphylococcus epidermidis, four $S$ warneri, three $S$ capitis, two $S$ hominis, one each of $S$ xylosus, $S$ simulans, $S$ equorum, and $S$ lugdunensis were identified. 37 CoNS were penicillin resistant, 12 gentamicin resistant, 28 tetracycline resistant, 18 erythromycin resistant, four ciprofloxacin resistant, and one teicoplanin resistant (MIC, $32 \mu \mathrm{g} / \mathrm{ml})$. In total, 16 strains were resistant to three or more antibiotics.

Conclusion-Species of CoNS apart from $S$ epidermidis may be isolated from patients with corneal and external infection. Antibiotic susceptibility of CoNS is unpredictable and multiresistant strains are common. As a result, antibiotic susceptibility testing should be performed in all cases of clinically significant ocular infections caused by CoNS.

(Br F Ophthalmol 1999;83:771-773)

Members of the genus Staphylococcus are nonmotile, non-spore forming, usually unencapsulated Gram positive cocci; most species are facultative anaerobes. They are indigenous microflora of the skin and mucous membranes of humans and are a common cause of eyelid, conjunctival, or corneal infection. ${ }^{1-7}$ The presence or absence of coagulase, an enzyme that clots plasma, divides the Staphylococcus species into two broad groups: the coagulase positive staphylococci and coagulase negative staphylococci (CoNS). Fourteen species of CoNS are currently recognised as human isolates. ${ }^{8}$ In the microbiology laboratory, identification of staphylococci is often limited to a rapid screening test for $S$ aureus, while non- $S$ aureus isolates are simply reported as CoNS species. Apart from being a common component of the normal ocular flora, these bacteria have also been reported to cause chronic blepharitis, ${ }^{23}$ conjunctivitis, ${ }^{45}$ and keratitis. ${ }^{67}$

Despite being important ocular pathogens, CoNS have so far received little attention in ophthalmology. The purpose of this study was to identify and determine antibiotic susceptibility of CoNS isolated from patients with chronic blepharitis, purulent conjunctivitis, and suppurative keratitis.

Materials and methods

A retrospective review of all culture positive cases of chronic blepharitis, purulent conjunctivitis, and suppurative keratitis between July 1995 and December 1996 was performed. All case in which CoNS were the sole isolates were selected. In total 55 clinically significant strains of CoNS from 45 outpatients were analysed. Thirty one CoNS were from eyes with chronic blepharitis, 12 from eyes with purulent conjunctivitis, and 12 from eyes with suppurative keratitis. Eight patients had bilateral chronic blepharitis and two bilateral acute conjunctivitis; in the remaining 35, ocular infection involved one eye only.

Specimens were collected from the inferior fornix of the palpebral conjunctiva, lid margins, and corneal ulcers using Dacron swabs. Three swabs were used for each eye. The first swab was inoculated into thioglycolate broth supplemented with sterile fetal calf serum $(10 \% \mathrm{vol} / \mathrm{vol})$ for the detection of aerobic and anaerobic bacteria. Tubes were incubated at $37^{\circ} \mathrm{C}$ for at least 72 hours. Subcultures were performed by using chocolate, mannitol salt, and McConkey agar plates. The second swab was rolled on a Sabouraud dextrose agar plate for the detection of fungi. The third swab was inoculated into 4SP transport medium for Chlamydia trachomatis. Cultures for the detection of bacteria, fungi, and $C$ trachomatis were performed according to a previously recommended protocol. ${ }^{9}$ CoNS were identified by Gram staining, colony morphology on agar plates, and slide test for coagulase. ${ }^{8}$

Over the 18 month period, as they were isolated, CoNS were immediately speciated. Species identification was performed by using the API ID 32 Staph (bioMérieux, La Balme les Grottes, Montalieu Vercieu, France), a stand- 
Table 1 Species identification of coagulase negative staphylococci isolated from patients with chronic blepharitis, purulent conjunctivitis, and suppurative keratitis

\begin{tabular}{llll}
\hline & \multicolumn{3}{l}{ No of cases } \\
\cline { 2 - 4 } $\begin{array}{l}\text { Species (No of } \\
\text { isolates) }\end{array}$ & $\begin{array}{l}\text { Chronic } \\
\text { blepharitis }\end{array}$ & $\begin{array}{l}\text { Purulent } \\
\text { conjunctivitis }\end{array}$ & $\begin{array}{l}\text { Suppurative } \\
\text { keratitis }\end{array}$ \\
\hline Sepidermidis (42) & 21 & 12 & 9 \\
S warneri (4) & 4 & - & - \\
S capitis (3) & 2 & - & 1 \\
S hominis (2) & 2 & - & - \\
Sxylosus (1) & - & - & - \\
S simulans (1) & 1 & - & - \\
S equorum (1) & 1 & - & 1 \\
S lugdunensis (1) & - & - & \\
\hline
\end{tabular}

ardised and miniaturised biochemical test system with a specially adapted database. The API ID 32 Staph system strip consists of 32 cupules, 26 of which contain dehydrated biochemical media for colorimetric tests. The tests included acid production from glucose, fructose, mannose, maltose, lactose, trehalose, mannitol, raffinose, sucrose, N-acetylglucosamine, turanose, ribose, arabinose, and cellobiose; decarboxylation of arginine and ornithine; production of urease, $\beta$ glucuronidase, $\beta$ galactosidase, acetoin, alkaline phosphatase, arginine arylamidase, and pyrrolidonyl-arylamidase; hydrolysis of aesculin; reduction of nitrate; and susceptibility to novobiocin. The manufacturer's recommended procedures were followed. Strain profiles were read and identified with automatic testing bacteriology equipment and were interpreted with APILAB software.

Antibiotic susceptibility tests were determined by agar disc diffusion (Kirby-Bauer method). ${ }^{10}$ The following antibiotics were tested: penicillin, gentamicin, tetracycline, erythromycin, ciprofloxacin, and teicoplanin. A standard agar dilution method ${ }^{11}$ was used to confirm teicoplanin resistance determined by agar disc diffusion. In brief, overnight cultures of bacteria $\left(\geq 10^{9}\right.$ colony forming units $/ \mathrm{ml}$ ) were plated at a series of dilutions on tryptic soy agar plates containing antibiotic-free medium or twofold dilutions of teicoplanin within the drug concentration range of 0.1-128 $\mu \mathrm{g} / \mathrm{ml}$. Plates were incubated at $37^{\circ} \mathrm{C}$ for 48 hours and the number of bacterial colonies was counted. Teicoplanin minimal inhibitory concentration (MIC) was defined as the lowest concentration of the antibiotic causing $99.9 \%$ loss of the bacterial inoculum. Teicoplanin MICs for most clinical isolates of CoNS have been reported to be $\leq 8 \mu \mathrm{g} / \mathrm{ml}$ or $\leq 16 \mu \mathrm{g} / \mathrm{ml}^{12}$

Table 2 Antibiotic susceptibility testing of coagulase negative staphylococci

\begin{tabular}{lllllll}
\hline Species & Penicillin & Gentamicin & Tetracycline & Erythromycin & Ciprofloxacin & Teicoplanin \\
\hline S epidermidis & $30 / 42^{\star}$ & $9 / 42$ & $20 / 36 \dagger$ & $14 / 42$ & $3 / 42$ & $1 / 42$ \\
S warneri & $3 / 4$ & $1 / 4$ & $3 / 4$ & $0 / 4$ & $0 / 4$ & $0 / 4$ \\
S capitis & $1 / 3$ & $1 / 3$ & $2 / 3$ & $2 / 3$ & $1 / 3$ & $0 / 3$ \\
S hominis & $1 / 2$ & $0 / 2$ & $2 / 2$ & $1 / 2$ & $0 / 2$ & $0 / 2$ \\
Sxylosus & $1 / 1$ & $1 / 1$ & $1 / 1$ & $1 / 1$ & $0 / 1$ & $0 / 1$ \\
S simulans & $0 / 1$ & $0 / 1$ & $0 / 1$ & $0 / 1$ & $0 / 1$ & $0 / 1$ \\
S equorum & $1 / 1$ & $0 / 1$ & $0 / 1$ & $0 / 1$ & $0 / 1$ & $0 / 1$ \\
Slugdunensis & $0 / 1$ & $0 / 1$ & $0 / 1$ & $0 / 1$ & $0 / 1$ & $0 / 1$ \\
Total & $37 / 55$ & $12 / 55$ & $28 / 49 \dagger$ & $18 / 55$ & $4 / 55$ & $1 / 55$ \\
\hline
\end{tabular}

*Numbers indicate resistant isolates/total isolates.

†Susceptibility to tetracycline was not performed in 6 isolates.
Table 3 Multiple antibiotic resistance of coagulase negative staphylococci

\begin{tabular}{lc}
\hline Resistance to & Number (\%) of isolates \\
\hline 2 antibiotics & $12(22)$ \\
3 antibiotics & $11(20)$ \\
4 antibiotics & $3(5)$ \\
5 antibiotics & $1(2)$ \\
6 antibiotics & $1(2)$ \\
\hline
\end{tabular}

Strains which showed multiple antibiotic resistance were stored in nutrient broth with $15 \%$ glycerol at $-80^{\circ} \mathrm{C}$ for further reference.

\section{Results}

Forty two $S$ epidermidis, four $S$ warneri, three $S$ capitis, two $S$ hominis, one each of $S$ xylosus, $S$ simulans, $S$ equorum, and $S$ lugdunensis were identified (Table 1). The results of antibiotic susceptibility testing are given in Tables 2 and 3. Overall, $37(67 \%)$ isolates were resistant to penicillin, $12(22 \%)$ to gentamicin, $18(33 \%)$ to erythromycin, four (7\%) to ciprofloxacin, one $(2 \%)$ to teicoplanin. Susceptibility to tetracycline was tested on 49 isolates; 28 (57\%) were found to be tetracycline resistant. On the whole, eight $(15 \%)$ strains were sensitive to all antibiotics, whereas 16 (29\%) were resistant to three or more antibiotics. One strain of $S$ epidermidis was found to be resistant to all antibiotics. Teicoplanin resistance determined by agar disc diffusion was confirmed by the agar dilution method (MIC $32 \mu \mathrm{g} / \mathrm{ml}$ ).

\section{Discussion}

Methods for the identification of CoNS include conventional as well as commercial systems. The conventional method ${ }^{8}$ is relatively cumbersome for routine laboratory use and may take up to 5 days of incubation to obtain a result. Commercial biochemical systems can identify a number of CoNS with accuracy of more than 95\% with relative speed and simplicity. ${ }^{13}$ In this study, species identification of CoNS was performed by using API ID 32 Staph, a commercially available biochemical test system which can identify 24 species. This system has been reported to be able to provide a correct species identification for $95.2 \%$ of CoNS analysed, with an accuracy of $98 \%-$ $100 \%$ for $S$ epidermidis, $S$ lugdunensis, $S$ capitis, $S$ warneri, and $S$ xylosus. ${ }^{13}$ In our study, eight species of CoNS were identified, the most common being $S$ epidermidis (76\% of all isolates). Other species of CoNS were identified in 10 patients with chronic blepharitis and in three with suppurative keratitis. Interestingly, all four isolates of $S$ warneri were from patients with chronic blepharitis. Strains of $S$ capitis, $S$ xylosus, and $S$ lugdunensis were isolated from patients with suppurative keratitis.

Because of their ubiquitous nature and relatively low virulence, staphylococci other than $S$ aureus are often simply reported as CoNS in the microbiology laboratory. However, over the past 15 years, there has been an increase in the documentation of ocular infections caused by CoNS. 
Evidence indicates that these bacteria play a primary role in the production of disease in clinical staphylococcal and mixed seborrhoeic/ staphylococcal blepharitis. ${ }^{3}$ In a relatively recent study, CoNS have been reported to be the most frequently isolated pathogens from patients with acute bacterial conjunctivitis. ${ }^{5}$ Two recent studies performed by the Bacterial Keratitis Study Research Group ${ }^{6}$ and the Ciprofloxacin Bacterial Keratitis Study Group ${ }^{7}$ have shown that the most frequent isolates from patients with bacterial keratitis were CoNS. Moreover, CoNS are currently the most common organisms isolated from cases of postoperative endophthalmitis. ${ }^{14} 15$

Because of their increasing importance, clinically significant CoNS should be identified to the species level. Species identification will improve our knowledge about the role played in clinical diseases by this group of bacteria.

In this study, CoNS isolated from patients with corneal and external infection showed highly variable patterns of antibiotic susceptibility. Eight $(15 \%)$ strains only were sensitive to all antibiotics. Most isolates were penicillin and tetracycline resistant, whereas resistance to ciprofloxacin and teicoplanin was found in four $(7 \%)$ and one $(2 \%)$, respectively. Given the high broad spectrum activity against most bacteria ${ }^{16}$ and the reduced frequency of ocular toxic effects, ${ }^{7}$ ciprofloxacin is currently considered the drug of choice in the therapy for bacterial keratitis. However, ophthalmologists should be aware that there is growing evidence for ciprofloxacin resistant strains of CoNS and should be prepared to modify treatment accordingly.

Teicoplanin, as well as vancomycin, is an anti-staphylococcal antibiotic to which resistance is rarely seen; their use should be reserved for the treatment of staphylococcal infections resistant to all other antibiotics. ${ }^{17}$

Multiple antibiotic resistance of CoNS is a well recognised problem, especially in nosocomial infections. ${ }^{12}{ }^{17}$ In this study, $16(29 \%)$ isolates were resistant to three or more antibiotics. Other authors ${ }^{2}$ reported resistance to two or more antibiotics in $25 \%$ of the staphylococcal isolates from patients with chronic blepharitis. Multiple antibiotic resistance might possibly represent a response to prolonged treatment. The detection of one strain resistant to all the antibiotics tested in this study is cause for concern. The spread of such strains in hospitals may constitute a threat for immunocompromised patients. Actually, multiresistant CoNS often colonise the skin of hospitalised patients and hospital personnel. ${ }^{12}$ Skin colonisation serves as a potential reservoir for multiresistant isolates that can cause infections.

To establish whether in vitro antimicrobial resistance of CoNS is clinically significant in the treatment of ocular infection is beyond the aims of this study. Other authors reported that treatment with ciprofloxacin in ciprofloxacin resistant $S$ epidermidis keratitis was unsuccessful. ${ }^{18}$ Similar results have been reported in the treatment of ciprofloxacin resistant Pseudomonas keratitis (Garg P, presented at the AAO Annual Meeting, New Orleans, 1998).

In conclusion, the need for species identification of clinically significant CoNS must be emphasised. With the availability of highly specific commercial identification systems, it is likely that species of CoNS apart from $S$ epidermidis will be increasingly implicated as causative organisms in corneal and external infection. Since the antibiotic susceptibility of CoNS is unpredictable and multiresistant isolates are common, we recommend to perform antibiotic susceptibility testing in all cases of clinically significant ocular infections caused by these organisms.

Presented in part as a poster at the American Academy Annual Meeting, San Francisco, California, 26-29 October 1997.

1 McCulley JP, Dougherty JM, Denau DG. Classification of chronic blepharitis. Ophthalmology 1982;89:1173-80.

2 Dougherty JM, McCulley JP. Comparative bacteriology of chronic blepharitis. Br f Ophthalmol 1984;68:524-8.

3 McCulley JP, Dougherty JM. Bacterial aspects of chronic blepharitis. Trans Ophthalmol Soc UK 1986;105:314-8.

4 Soukiasian SH, Baum J. Bacterial conjunctivitis. In: Krachmer JH, Mannis MJ, Holland EJ, eds. Cornea. St Louis, Mosby-Year Book, 1996; vol 2: chap 63.

5 Leibowitz HM. Antibacterial effectiveness of ciprofloxacin $0.3 \%$ ophthalmic solution in the treatment of bacterial conjunctivitis. Am f Ophthalmol 1991;112:29S-33S.

6 O'Brien TP, Maguire MG, Fink NE, et al. Efficacy of ofloxacin vs cefazolin and tobramycin in the therapy for bacterial keratitis. Arch Ophthalmol 1995;113:1257-65.

7 Hyndiuk RA, Eiferman RA, Caldwell DR, et al. Comparison of ciprofloxacin ophthalmic solution $0.3 \%$ to fortified

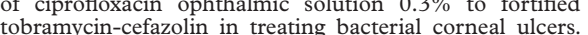
tobramycin-cefazolin in treating
Ophthalmology 1996;103:1854-63.

8 Kloos WE, Bannerman TL. Staphylococcus and MicrococKloos WE, Bannerman TL. Staphylococcus and Micrococ-
cus. In: Murray PR, Baron EJ, Pfaller MA, Tenover FC, Yolken RH, eds. Manual of clinical microbiology. 6th ed. Washington, DC: American Society for Microbiology, 1995: chap 22.

9 Jones DB, Liesegang TJ, Robinson NM. Cumitech 13: laboratory diagnosis of ocular infections. Washington, DC: American Society for Microbiology, 1981

10 National Committee for Clinical Laboratory Standards. Performance standards for antimicrobial disk susceptibility tests. Approved standards M2-A5. Villanova, PA: National Committee for Clinical Laboratory Standards, 1993.

11 National Committee for Clinical Laboratory Standards. Methods for dilution antimicrobial susceptibility tests for bacteria that grow aerobically. Approved standard M7-A4. Villanova, PA: National Committee for Clinical Laboratory Standards, 1997 .

12 Archer GL, Climo MW. Antimicrobial susceptibility of coagulase-negative staphylococci. Antimicrob Agents Chemother 1994;38:2231-7.

13 Ieven M, Verhoeven J, Pattyn SR, et al. Rapid and economical method for identification of clinically significant coagulase-negative staphylococci. f Clin Microbiol 1995;33: $1060-3$

14 Kattan HM, Flynn HW, Pfugfelder SC, et al. Nosocomial endophthalmitis survey: current incidence of infection after surgery. Ophthalmology 1991;98:227-38.

15 Speaker MG, Milch FA, Shah MK, et al. Role of external bacterial flora in the pathogenesis of acute postoperative endophthalmitis. Ophthalmology 1991;98:639-49.

16 Neu HC. Microbiologic aspects of fluoroquinolones. $A m \mathcal{F}$ Ophthalmol 1991;112:15S-24S.

17 Degener JE, Heck MEOC, van Leeuwen WJ, et al. Nosocomial infection by Staphylococcus haemolyticus and typing methods for epidemiological study. $\mathcal{F}$ Clin Microbiol 1994;32:2260-5.

18 Snyder ME, Katz HR. Ciprofloxacin-resistant bacterial keratitis. Am $\mathcal{f}$ Ophthalmol 1992;114:336-8. 